\title{
Analytical Study of Task Offloading Techniques using Deep Learning
}

\author{
Almelu \\ PhD Scholar \\ Computer Science \& Engineering, \\ Rabindranath Tagore University \\ Bhopal, Madhya Pradesh, India \\ almelu02@gmail.com
}

\author{
Dr. S. Veenadhari, \\ Associate Professor \\ Computer Science \& Engineering, \\ Rabindranath Tagore University \\ Bhopal, Madhya Pradesh, India
}

\author{
Kamini Maheshwar \\ $\mathrm{PhD}$ Scholar \\ Computer Science \& Engineering, \\ Rabindranath Tagore University \\ Bhopal, Madhya Pradesh, India
}

\begin{abstract}
The Internet of Things (IoT) systems create a large amount of sensing information. The consistency of this information is an essential problem for ensuring the quality of IoT services. The IoT data, however, generally suffers due to a variety of factors such as collisions, unstable network communication, noise, manual system closure, incomplete values and equipment failure. Due to excessive latency, bandwidth limitations, and high communication costs, transferring all IoT data to the cloud to solve the missing data problem may have a detrimental impact on network performance and service quality. As a result, the issue of missing information should be addressed as soon as feasible by offloading duties like data prediction or estimations closer to the source. As a result, the issue of incomplete information must be addressed as soon as feasible by offloading duties such as predictions or assessment to the network's edge devices. In this work, we show how deep learning may be used to offload tasks in IoT applications.
\end{abstract}

Keywords- Internet of Things (IoT), Bandwidth, Task Offloading, Deep Learning.

\section{INTRODUCTION}

For each IoT network, some terminals carry out their activities locally, while other terminals simultaneously outsource their activities in the gateway. At the beginning of each time period, each terminal decides to perform tasks and transfer power. The Internet of Things (IoT) is a new paradigm that integrates a large number of sensor-based elements such as devices, sensors and actuators on the Internet. These elements constantly generate huge amounts of data with many characteristics such as large, heterogeneous, missing, corrupt and noisy. The quality and quantity of this data are critical issues for intelligent data processing and analysis for IoT applications. However, during data collection, missing data is encountered due to various reasons such as: B. Unstable network communication, timing issues, device errors, and manual shutdown. This missing and corrupted data impacts the reliability, scalability, and interoperability of applications causing incorrect conclusions and decisions. Indeed, this problem can lead to catastrophic results [3].

\section{APPLICATION OF IOT}

Wearable Devices: Virtual glasses, fitness bracelets for tracking calorie consumption and heart rate, for example, or GPS tracking belts, are just a few examples of wearable devices we've been using for some time. Companies like Google, Apple, Samsung and others have developed and introduced the Internet of Things and its application in our daily life.

Health: Using wearable devices or sensors connected to patients allows doctors to monitor a patient's condition outside the hospital and in real time. By constantly monitoring certain measurements and automatically alerting them of their vital signs, the Internet of Things helps improve patient care and prevent fatal events in high-risk patients.

Another area of application is the integration of IoT technology in hospital beds, which are being replaced by smart beds equipped with special sensors to monitor vital signs, blood pressure, oximeters and body temperature, among others.

Traffic Monitoring: The Internet of Things can be very useful for controlling vehicular traffic in large cities and can contribute to the concept of smart cities.

Fleet management: The installation of sensors in fleet vehicles enables efficient networking between vehicles and their managers, as well as between vehicles and their drivers. Both the driver and the manager / owner can know every type of detail about the state, operation and needs of the vehicle simply by accessing the software for collecting, processing and organizing data. You even get real-time alerts on maintenance incidents without the operator knowing.

Agriculture: Smart farms are a given. Soil quality is essential to producing good harvests, and the Internet of Things gives farmers the opportunity to access detailed knowledge and valuable information about their soil conditions. Using IoT sensors, it is possible to obtain considerable data on soil conditions and stages. Information such as soil moisture, acidity, presence of certain nutrients, temperature and many other chemical properties help farmers control irrigation, make water use more efficient, determine the best time to start sowing and even the presence of diseases in plants and soil. 
Hospitality: The application of the IoT in the hospitality sector brings interesting improvements in the quality of service. Locating customers, sending offers or information on interesting activities, fulfilling orders for room or room service, automatically debiting the room bill or requesting toiletries are activities that are carried out through applications integrated with Internet technology. Objects are easily managed.

Smart grid and energy saving: The progressive use of intelligent or sensor-equipped energy meters and the installation of sensors in various strategic points leading from the production plants to the various distribution points allow for better monitoring and control of the electricity grid.

Maintenance management: One of the areas where the application of IoT technology is most extensive is precisely maintenance management. Through the combination of sensors and software specialized in CMMS/EAM maintenance management, a multifunctional tool is obtained whose use can be applied to a multiplicity of disciplines and practices, with the purpose of extending the useful life of physical assets, while guaranteeing asset reliability and availability.

\section{RELATED WORK}

\section{A. Distributed Deep Learning Driven Task Offloading (DDTO) Algorithm for IoT}

The DDTO algorithm determines whether a task should be executed at a local device or whether it should be offloaded to the clouds, and if it should be offloaded to the clouds the algorithm determines, whether to offload the task to the central cloud or to the edge cloud. The numerical results demonstrate the accuracy of DDTO algorithm and in comparison, with several previously known schemes results are significantly better [1].

\section{B. Deep Learning Based Missing Data Prediction Protocol (DEEPMDP) For IoT}

DeepMDPt solves the missing sensor data problem in IoT via DL models. Considering a three-layered IoT network, DeepMDP is designed to run on all three layers. DeepMDP adaptively predicts any missing value in received sensor values from lower layers of the IoT network and transforms them to the compact format in a size-efficient way. DeepMDP checks whether there is missing data in the received raw weather data, if missing data exists, it predicts and completes the missing data, and sends it to the subscribers in the form of Spatio-temporal weather pattern (STWP). Here, STWP refers to a significant pattern formed from sensor values collected from a specific location. With this approach, DeepMDP eliminates the missing data problem and transmits the data to the subscribers in more compact STWP format instead of sending all the raw sensor data, thus contributes to the reduction of the network data transmission load [3].

Offload calculations for IoT in C-RAN Offload calculations for Internet of Things (IoT) applications in the Cloud Radio Access Network (CRAN) Multiple Input Multiple Output (MIMO). In particular, the computational activities of IoT devices (IoTD) are subcontracted to a MIMO C-RAN, where a MIMO radio resource head ( $\mathrm{RRH})$ is connected to a baseband unit (BBU) via a connection front panel with limited capacity., facilitated by spatial filtering and uniform scalar quantization. A compute offload optimization problem is formulated to minimize the overall transmission power of the IoTDs and at the same time meet the latency requirements of compute tasks. In order to obtain a workable solution for the non-convex problem, the spatial filter matrix is first optimized locally on MIMO RRH. Then, using the alternative optimization framework for joint optimization of residual variables at the BBU level, the baseband combiner, optimal resource allocation, and number of quantization bits are determined by the minimum root mean square error metric ( MMSE), the next internal convex method or row search method [4].

\section{Space/Aerial-Assisted Computing Offloading for IoT Applications: A Learning-based Approach}

The IoT computing of flooding problem in SAGIN, they have proposed a joint VM allocation and task scheduling mechanism to efficiently allocate the computing resources to different VMs in the UAV edge server. To offload the computation-intensive tasks, we have proposed an RLbased computing offloading approach to handle the multidimensional SAGIN resources and learn the dynamic network conditions. Deep neural networks, policy gradient, and actor-critic methods have been employed to improve the learning performance. Simulation results have validated the convergency and efficiency of the proposed approaches. [5].

\section{Offloading for Energy Harvesting IoT Devices via Policy-based Deep Reinforcement Learning}

Policy-based deep reinforcement learning algorithm to optimize the time and workload allocation in a novel backscatter-assisted hybrid MEC offloading scenario. Comparing with the value-based deep Q-network algorithm, the policy-based deep deterministic policy gradient method achieves significant improvement in terms of reward performance, stability, and the learning speed[7].

\section{E. Deep Reinforcement Learning Approach for Collaborative Mobile Edge Computing IoT Networks}

AI based task allocation algorithm (iRAF) has been proposed for the collaborative mobile edge computing network. iRAF is trained by self-play policy without manually collecting data and labels. The proposed algorithm could sense the changing of network environment and adaptively update the resource allocation strategy. Since the core algorithm of iRAF, MCTS with a deep neural network, has a strong capability to solve the problem with complex action space, it exhibits higher performance than deep Q learning based DRL algorithm, as example of AlphaGo. The simulation results prove that iRAF has better performance on the average latency and energy consumption than the existing main methods. Besides, iRAF can further improve the performance by adding a prediction neural network in our future work they believe that the $\mathrm{iRAF}$ exhibits a promising application prospective in the massively interconnected and computation-intensive IoT scenarios [8]. 


\section{F. Task Proactive Caching Based Computation Offloading and Resource Allocation in Mobile-Edge Computing Systems}

Compute offloading, resource allocation, and proactive process caching are all optimized together to minimize execution latency under the constraints of radio, compute, and storage resources. To solve the MINLP problem, they offer a proactive caching algorithm for collaboration between the cloud and the MEC server to determine the caching status of the job. Next, we propose a three-step heuristic algorithm based on a greedy strategy to solve the allocation of resources and the selection of the execution mode of the tasks [9].

\section{G. Learning-based Computation Offloading for IoT Devices with Energy Harvesting}

Energy harvesting powered mobile edge computing is a promising approach to improve the computation capabilities for self-sustainable IoT devices. The RL-based computation offloading framework for IoT devices with EH to achieve the optimal offloading policy without awareness of the MEC model, the computation latency and energy consumption model. An RLO scheme has been proposed for IoT devices with low complexity, which utilizes the transfer learning technique to accelerate the learning speed. We have also presented a DRLO scheme that uses CNN to compress the state space in the learning process. Furthermore, the conditions for both "fully offloading" and "locally processing" are provided, and the performance bounds of the proposed RL-based offloading after convergence under three typical scenarios are studied. Simulations have been performed for the IoT device with RF signal-based wireless power transfer to verify the theoretical results, indicating that the DRLO scheme generates the best policy, and outperforms the DRL scheme with $58.3 \%$ lower energy consumption, $26.7 \%$ less computation latency, and 55.5\% lower task drop rate [10].

\section{H. Collaborative Task Offloading Mechanism for Mobile Cloudlet Networks}

This paper has investigated the load balancing problem in mobile cloudlet networks. By leveraging balls-and-bins theory, they have devised 'CTOM', a collaborative task offloading scheme for mobile cloudlet networks. By locally querying limited task load information, the proposed solution can reduce the longest task queue in the allocation process effectively. simulation and trace-driven evaluation results have demonstrated that CTOM performs exceedingly close to the optimal solution in load balancing and outperforms the conventional random and proportional allocation schemes by $65 \%$ and $55 \%$ respectively in task gaps, while its computing complexity has been largely reduced at each time interval [11].

Table I. Gives the Comparative Study of Various Methods for Task Offloading in IOT

\begin{tabular}{|l|l|l|l|}
\hline Author & Description & Results & Drawback \\
\hline
\end{tabular}

\begin{tabular}{|c|c|c|c|}
\hline $\begin{array}{l}\text { Huaming } \\
\text { Wu et al. } \\
(2020)\end{array}$ & $\begin{array}{l}\text { Distributed } \\
\text { Deep } \\
\text { learning- } \\
\text { driven Task } \\
\text { Offloading } \\
\text { (DDTO) } \\
\text { algorithm }\end{array}$ & $\begin{array}{l}\text { Effectively and } \\
\text { efficiently } \\
\text { generate near- } \\
\text { optimal } \\
\text { offloading } \\
\text { decisions in } \\
\text { edge and cloud } \\
\text { computing } \\
\text { environments }\end{array}$ & $\begin{array}{l}\text { Data privacy } \\
\text { and security } \\
\text { problems }\end{array}$ \\
\hline $\begin{array}{l}\text { Yi Liu et } \\
\text { al. } \\
(2020)\end{array}$ & $\begin{array}{l}\text { Mobile Edge } \\
\text { Computing in } \\
\text { Power IoT } \\
\text { System }\end{array}$ & $\begin{array}{l}\text { It provides } \\
\text { computation } \\
\text { services for } \\
\text { both local } \\
\text { devices and } \\
\text { neighboring } \\
\text { small-cells. } \\
\end{array}$ & $\begin{array}{l}\text { Computation } \\
\text { problem in both } \\
\text { centralized and } \\
\text { distributed } \\
\text { system. }\end{array}$ \\
\hline $\begin{array}{l}\text { I’brahim } \\
\text { Ko“k et } \\
\text { al. } \\
(2020)\end{array}$ & $\begin{array}{l}\text { DeepMDP for } \\
\text { IOT }\end{array}$ & $\begin{array}{l}\text { Reduces } \\
\text { network load } \\
\text { and latency }\end{array}$ & $\begin{array}{l}\text { Bandwidth } \\
\text { limitation and } \\
\text { high } \\
\text { communication } \\
\text { cost }\end{array}$ \\
\hline $\begin{array}{l}\text { Chandan } \\
\text { Pradhan } \\
\text { et al. } \\
(2020)\end{array}$ & $\begin{array}{l}\text { Computation } \\
\text { Offloading } \\
\text { for IoT in C- } \\
\text { RAN }\end{array}$ & $\begin{array}{l}\text { Minimizes the } \\
\text { total transmit } \\
\text { power of the } \\
\text { IoTDs }\end{array}$ & \\
\hline $\begin{array}{l}\text { Nan } \\
\text { Cheng et } \\
\text { al. } \\
(2019)\end{array}$ & $\begin{array}{l}\text { Space/Aerial- } \\
\text { Assisted } \\
\text { Computing } \\
\text { Offloading } \\
\text { for IoT } \\
\text { Applications } \\
\end{array}$ & $\begin{array}{l}\text { Converges fast, } \\
\text { but also } \\
\text { achieves a } \\
\text { lower total cost. }\end{array}$ & $\begin{array}{l}\text { optimizing the } \\
\text { communication, } \\
\text { caching, and } \\
\text { computing } \\
\text { resources }\end{array}$ \\
\hline $\begin{array}{l}\text { Jienan } \\
\text { Chen et } \\
\text { al. } \\
(2019)\end{array}$ & $\begin{array}{l}\text { iRAF fo } \\
\text { Mobile Edge } \\
\text { Computing } \\
\text { IoT Networks }\end{array}$ & $\begin{array}{l}\text { Better } \\
\text { performance on } \\
\text { the average } \\
\text { latency and } \\
\text { energy } \\
\text { consumption }\end{array}$ & \\
\hline $\begin{array}{l}\text { Minghui } \\
\text { Min et } \\
\text { al. } \\
(2018)\end{array}$ & $\begin{array}{l}\text { Mobile edge } \\
\text { computing } \\
\text { and energy } \\
\text { harvesting } \\
(\mathrm{EH})\end{array}$ & $\begin{array}{l}\text { Reduces the } \\
\text { energy } \\
\text { consumption, } \\
\text { computation } \\
\text { latency and task } \\
\text { drop rate }\end{array}$ & \\
\hline
\end{tabular}

IV. CONCLUSION

In this paper, we studied different technique used for deep learning in IOT application. (DDTO) algorithm used for cloud computing. Edge Computing provide computation services for both local devices and neighboring small-cells. DeepMDP reduce network load. C-RAN Minimizes the total transmit power of the IoTDs. Space/Aerial-Assisted Computing Offloading. Converges fast, but also achieves a lower total cost. iRAF to Mobile Edge Computing provide better performance on the average latency and energy consumption. energy harvesting (EH) Reduces the energy consumption, computation latency and task drop rate.

\section{REFERENCES}

[1] Huaming Wu, Member, IEEE, Ziru Zhang, Chang Guan, Katinka Wolter, and Minxian Xu, " Collaborate Edge and Cloud Computing with Distributed Deep Learning for Smart City" in IEEE Internet of Things Journal, 2020.

[2] Yi Liu, Shengli Xie, Fellow, IEEE, and Yan Zhang, Fellow, IEEE Transactions on Vehicular Technology" Cooperative Offloading and Resource Management for UAV-Enabled Mobile Edge Computing in Power IoT System" IEEE Transactions on Vehicular Technology, August 18,2020

[3] 'Brahim and Suat O" zdemir "DeepMDP: A Novel Deep Learning Based Missing Data Prediction Protocol for IoT", IEEE Internet of Things Journal, 2020. 
[4] Chandan Pradhan, Student Member, IEEE, Ang Li, Member, IEEE et al" Computation Offloading for IoT in C-RAN Optimization and Deep Learning", IEEE Transactions on Communications, June 02, 2020.

[5] Nan Cheng, Member, IEEE, Feng Lyu, Member et al, "Space/Aerial-Assisted Computing Offloading for IoT Applications: A Learning-based Approach", in IEEE journal, 2020.

[6] Xiaolan Liu, Zhijin Qin al et," Resource Allocation for Edge Computing in IoT Networks via Reinforcement Learning," in IEEE, 2019.

[7] Yutong Xie Zhengzhuo $\mathrm{Xu}$ al et. "Backscatter-Assisted Computation Offloading for Energy Harvesting IoT Devices via Policy-base Deep Reinforcement Learning", IEEE/CIC International Conference on Communications Workshops in China, 2019 .

[8] Jienan Chen, Member Siyu Chen al et, "iRAF: a Deep Reinforcement Learning Approach for Collaborative Mobile Edge Computing IoT Networks," IEEE INTERNET OF THINGS JOURNAL, MONTH 2019.

[9] Hongyu Zhao, Ying Wang al et. "Task Proactive Caching Based Computation Offloading and Resource Allocation in Mobile-Edge Computing Systems", in IEEE, 2018.

[10] Minghui Min,Liang Xiao al et, "Learning-based Computation Offloading for IoT Devices with Energy Harvesting”, IEEE Transactions on Vehicular Technologyon, 2018.

[11] Xiaochen Fan, Xiangjian He al et, "CTOM: Collaborative Task Offloading Mechanism for Mobile Cloudlet Networks" IEEE. 2018. 